\title{
Breaking the Diffraction Barrier in Fluorescence Microscopy by Optical Shelving
}

\author{
Stefan Bretschneider, Christian Eggeling, and Stefan W. Hell ${ }^{*}$ \\ Department of NanoBiophotonics, Max Planck Institute for Biophysical Chemistry, 37070 Göttingen, Germany
}

(Received 31 January 2007; published 24 May 2007)

\begin{abstract}
We report the breaking of the diffraction resolution barrier in far-field fluorescence microscopy by transiently shelving the fluorophore in a metastable dark state. Using a relatively modest light intensity of several $\mathrm{kW} / \mathrm{cm}^{2}$ in a focal distribution featuring a local zero, we confine the fluorescence emission to a spot whose diameter is a fraction of the wavelength of light. Nanoscale far-field optical resolution down to $50 \mathrm{~nm}$ is demonstrated by imaging microtubules in a mammalian cell and proteins on the plasma membrane of a neuron. The presence of dark states in virtually any fluorescent molecule opens up a new venue for far-field microscopy with resolution that is no longer limited by diffraction.
\end{abstract}

DOI: 10.1103/PhysRevLett.98.218103

The discovery of the diffraction resolution barrier by Abbe in 1873 [1] has lead to the widely accepted notion that the resolution of a far-field light microscope is limited to about half of the wavelength of light, $\lambda / 2$. Since the mid 1980 s, near-field optics have been developed as a stylusbased alternative, but this approach is limited to imaging surfaces. Being noninvasive, far-field light microscopy remained the most popular microscopy mode in the life sciences, with fluorescence being its most important readout. Fortunately, the fluorescence contrast mode is favorable to overcome Abbe's barrier, since changing the parameters of fluorescence emission allows one to surmount the limiting role of diffraction.

For example, stimulated emission depletion (STED) microscopy breaks the diffraction barrier by inhibiting fluorescence emission of the fluorescent marker [2]. Other molecular processes for "switching off" the fluorescence are optically saturable conformational changes, such as photoinduced cis-trans isomerization and photoinduced cyclization reactions; they can be exploited similarly to STED $[3,4]$. Breaking the diffraction barrier is also possible by switching the fluorescence "on, " both in molecular ensembles [5,6] and on a single molecule level $[7,8]$. However, all these molecular mechanisms and strategies have their own strengths and limitations. Stimulated emission calls for comparatively large beam intensities of $10^{8}$ to $10^{10} \mathrm{~W} / \mathrm{cm}^{2}$, while photoswitching is restricted to a subset of fluorophores. Exploring alternative molecular mechanisms that combine these advantages is of utmost importance for establishing far-field optical nanoscopy.

In this Letter, we break the diffraction resolution barrier by shelving the fluorophore in a metastable dark state, thereby effectively depleting the fluorophore's ground state $S_{0}$. Optical shelving with a focal intensity distribution featuring an intensity zero transiently confines the fluorescence emission to spots of size $\ll \lambda$ at the sample. Scanning such transiently created spots readily yields subdiffraction images from the sample. While the experiments reported herein exhibit a resolution down to $50 \mathrm{~nm}$, our approach is inherently not limited by diffraction, and contrary to previously reported subdiffraction resolution tech-
PACS numbers: 87.64.Tt, 33.50.Dq, 42.30.-d, 33.50.-j

niques, it relies both on modest continuous wave (cw) illumination intensities and on standard fluorescent markers.

A primary candidate for optical shelving is the lowest (dark) triplet state $T_{1}$ of the molecule [Fig. 1(a)], first, because it is a characteristic of all fluorophores and, second, because it is metastable. Optical transitions between singlet and triplet states require a spin flip and hence are optically suppressed [9]. Nevertheless, effectively populating the $T_{1}$ is possible by repetitively exciting the dye to the first excited singlet state $S_{1}$. This is because on every excitation event from the singlet ground state $S_{0}$ to the fluorescent $S_{1}$, there is a finite probability of a nonradiative intersystem crossing $S_{1} \rightarrow T_{1}$. Featuring a lifetime of $\tau_{T}>$ $1-10^{4} \mu \mathrm{s}, T_{1}$ becomes saturated by excitation rates $k_{\mathrm{exc}}=$ $\sigma I_{D} /(\hbar \omega) \gg\left(\tau_{T}\right)^{-1}$. With typical ground state absorption cross sections of $\sigma \sim 10^{-16} \mathrm{~cm}^{2}$ and photon energies of $\hbar \omega=(2-4) \times 10^{-19} \mathrm{Ws}$, fluorescence depletion is readily attained at comparatively low intensities $I_{D}=10^{3}$ to $10^{5} \mathrm{~W} / \mathrm{cm}^{2}$ [Fig. 1(a)].

Implementing this concept is obviously challenged by the involvement of dark states in photobleaching $[10,11]$ which accounts for the fact that, although ground state depletion (GSD) microscopy was suggested more than a decade ago $[12,13]$, breaking the diffraction barrier by $T_{1}$ pumping has not been pursued. Our results became possible by discovering conditions at which $\mu$ s- to ms-long optical shelving is practical.

To establish these conditions, we performed pumpprobe experiments in a confocalized stage-scanning microscope with a standard 1.4 numerical aperture (NA) oil immersion lens (Leica Microsystems, Germany). Both the pump (i.e., depletion) and the probe beam originated from the same laser (Verdi, Coherent, CA) emitting a cw beam at $\lambda=532 \mathrm{~nm}$. The beams excited Atto532, a rhodamine-like fluorophore with maximum emission at $\sim 560 \mathrm{~nm}$. However, whereas the stronger pump beam was used to deplete $S_{0}$, the weaker probe beam measured the residual $S_{0}$ population [Fig. 1(b)]. Pumping and subsequent probing was realized by modulating the intensity of the beams with an acousto-optic modulator. The 

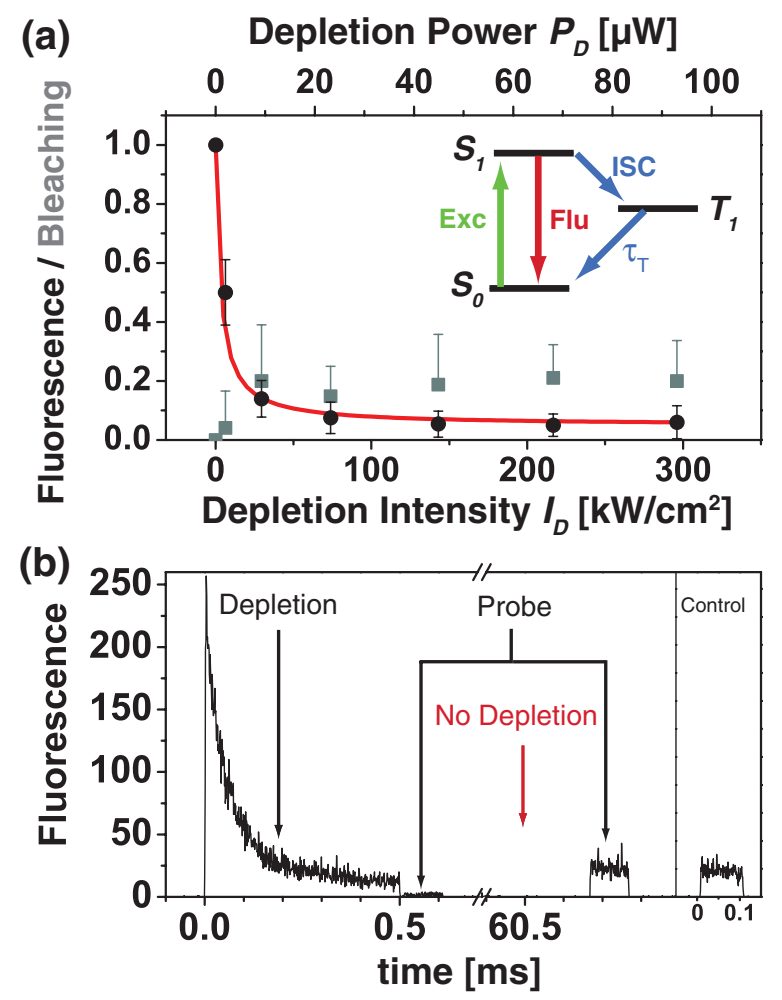

FIG. 1 (color). Fluorescence depletion of the organic dye Atto532 by triplet state $\left(T_{1}\right)$ buildup. (a) Fluorescence (black) and fraction of bleached molecules after 25 depletion-probe cycles (gray) as a function of depletion intensity $I_{D}$ or power $P_{D}$; error bars as relative standard deviation from 5 consecutive measurements. Inset: Fluorescence depletion is induced by $T_{1}$ buildup. (b) Depletion-probe cycle with strong depletion illumination $\left(500 \mu \mathrm{s}, I_{D}=85 \mathrm{~kW} / \mathrm{cm}^{2}\right)$, depleting the fluorescence due to $T_{1}$ buildup; subsequent low-power illumination (100 $\mu \mathrm{s}$, $I_{P}=7 \mathrm{~kW} / \mathrm{cm}^{2}$ ) probing the residual fluorescence signal. After depletion and complete relaxation of the $T_{1}$ ( $\sim 60 \mathrm{~ms}$ ), fluorescence emission is completely recovered, as observed by comparison with the signal gained just with the probe beam (Control).

Atto532 molecules were bound to antibodies (antimouse IgG, Jackson ImmunoResearch Laboratories) and mounted in Vectashield (Vector Laboratories, Burlingame, CA) in order to have the same chemical environment as in immunofluorescence imaging. Under these conditions, the fluorescence completely recovered $60 \mathrm{~ms}$ after depletion. We can assume without loss of generality that the observed dark state is the $T_{1}$ or another dark state that is populated through the $T_{1}$.

A recovery of the fluorescence is important, because our concept relies on scanning. In other words, the $T_{1}$ fluorophores need to relax to $S_{0}$ before the beam moves on. We thus have to balance the shelving time $\tau_{T}$, the intersystem crossing rate $k_{\mathrm{ISC}}$, and the photobleaching activity. Following a common 3-level system of a dye [Fig. 1(a)], the steady-state $T_{1}$ population relative to the singlet system $\left(S_{0}+S_{1}\right)$ population is $k_{\mathrm{ISC}} \tau_{T}\left[\left(\sigma I_{D} /(\hbar \omega)\right) /\left(k_{10}+\right.\right.$ $\left.\sigma I_{D} /(\hbar \omega)\right)$ ], with $k_{10}$ denoting the rate for spontaneous
$S_{1} \rightarrow S_{0}$ relaxation. Large $k_{\text {ISC }}$ and long $\tau_{T}$ yield efficient $T_{1}$. However, these parameters should not be too large. Longer $\tau_{T}$ entails longer image acquisition times. High intersystem crossing rates $k_{\text {ISC }}$ reduce the fluorescence quantum yield. With typical $\sigma \approx 10^{-16} \mathrm{~cm}^{2}$ and $k_{10} \approx$ $5 \times 10^{8} \mathrm{~s}^{-1}$, one readily finds that, for $k_{\mathrm{ISC}} \tau_{T}>100$ and depletion intensities as low as $I_{D}=10 \mathrm{~kW} / \mathrm{cm}^{2}$, the fluorescence is depleted by half, and rather modest values of $I_{D}>50 \mathrm{~kW} / \mathrm{cm}^{2}$ deplete the ground state to $<10 \%$ without exacerbating bleaching. Moreover, $\tau_{T}, k_{\mathrm{ISC}}$, and the photostability can be tuned by changing the environmental conditions. For example, $\tau_{T}$ can be increased by decreasing the concentration of molecular oxygen through sample embedding media that contains oxygen scavengers, such as Vectashield. In fact, we found Vectashield to be appropriate for GSD because, as shown in Fig. 1, we readily depleted the fluorescence down to $10 \%$ while still having a substantial fluorescence flux during probing. We repeated the depletion-probe cycle 25 times with only $20 \%$ photobleaching; $\tau_{T} \approx 10 \mathrm{~ms}$ allowed for a reasonable pixel dwell time of $60 \mathrm{~ms}$.

Next, we engineered the focal intensity distribution of the depletion beam to feature a central minimum by modifying the phase in a plane conjugate to the entrance pupil of the objective lens, using a spatial phase modulator (Hamamatsu, Japan). For the depletion spot with the $x$-oriented line-shaped minimum [Fig. 2(a)], we applied a phase shift of $\pi$ in a semiarea of the $x$-polarized depletion wave front entering the lens. Alternatively, we produced a doughnut [Fig. 2(b)] by imprinting a helical phase ramp $\exp (i \varphi)$ with $0 \leq \varphi \leq 2 \pi$ on a circularly polarized wave front. Exploiting a reversible saturable fluorescence depletion transition and a focal spot featuring central zeros, the resolution $d$ of GSD microscopy obeys [3]

$$
d \approx \lambda /\left[2(\mathrm{NA}) \sqrt{1+I_{D}^{\max } / I_{S}}\right],
$$

with $I_{S}$ denoting the saturation intensity at which half of the fluorescence is depleted and $I_{D}^{\max }$ is the maximum value of the peak bordering the zero. In conjunction with the depletion curve of Fig. 1(a), the depletion focal spots of Fig. 2 assure that $T_{1}$ molecules are built up everywhere in the focal region, except at the line-shaped or pointlike central minimum. Equation (1) implies that by increasing $I_{D}^{\max } \gg I_{S}$ fluorescence emission is confined to subdiffraction dimensions $d \ll \lambda / 2$, from where the signal is readily obtained by an overlaid probe beam.

While the line-shaped depletion spot improves the resolution along the $\mathrm{x}$ axis only, the doughnut-shaped spot acts in all lateral directions of the focal plane (Fig. 2). The effective, subdiffraction sized fluorescence focal spots, i.e., the effective point-spread functions (PSF) of the system, were calculated using the experimentally determined depletion and probe spots, in combination with the depletion curve of Fig. 1(a). Their full width at half maxima (FWHM) of the effective PSF decreases with increasing $I_{D}^{\max }[14]$. While providing an improved resolution along 


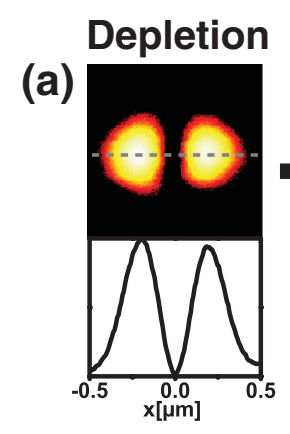

(b)
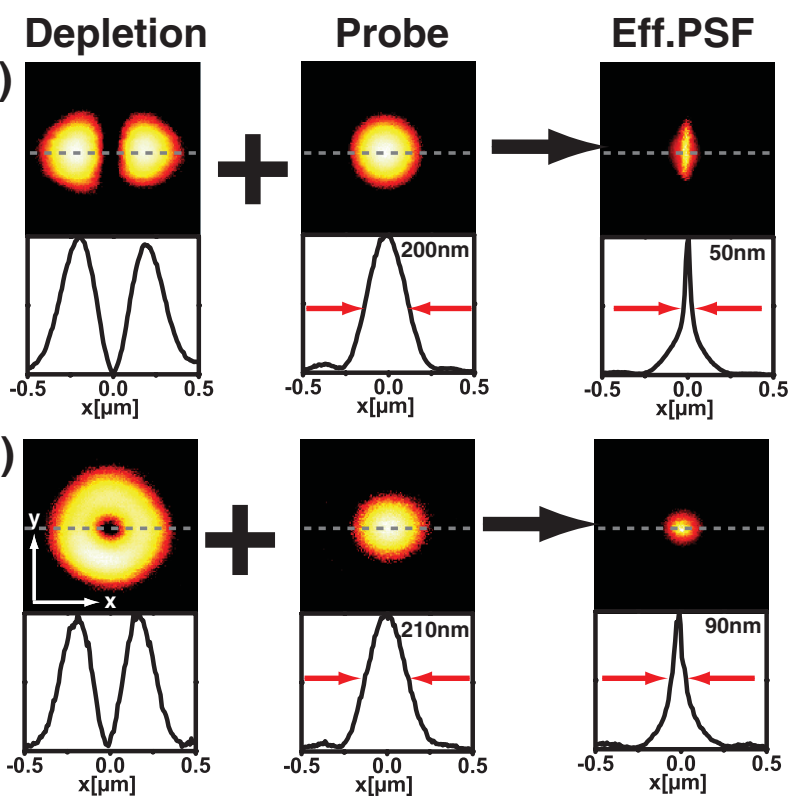

FIG. 2 (color). Creating effective PSFs of subdiffraction extent in a single point scanning GSD microscope. (a) Squeezing the spot just along the $\mathrm{x}$ axis and (b) along all directions in the focal plane. The depletion spot (Depletion) overlaid with the regularly focused probe spot (Probe) produces the effective PSF (Eff. PSF). The probe spot probes the fluorescence right after the depletion spot has pumped the dye into the $T_{1}$. Focal plane cross section of the PSF (upper panel) and profiles along the $\mathrm{x}$ axis [dashed line in upper panels] showing FWHM values (lower panels). Wavelengths for depletion and probing: $532 \mathrm{~nm}$; fluorescence wavelength: $560 \mathrm{~nm}$.

all lateral directions, the doughnut necessitates more scanning steps. To avoid increased photobleaching, we applied only $I_{D}^{\max }=80 \mathrm{~kW} / \mathrm{cm}^{2}$ in the doughnut case as compared to $I_{D}^{\max }=350 \mathrm{~kW} / \mathrm{cm}^{2}$ for the line. The $I_{D}^{\max } / I_{S}=35$ for the line and $I_{D}^{\max } / I_{S}=8$ for the doughnut rendered 50 and $90 \mathrm{~nm}$ FWHM, respectively. These FWHM values are slightly larger than those expected from Eq. (1), which is due to adverse effects such as $T_{1}$ relaxation during probing and imperfections of the zero [14]. In any case, the effective PSF is up to 4 times smaller than that of the confocal PSF resulting from the probe spot. Scanning the subdiffraction PSFs across the sample renders images with subdiffraction resolution.

Figure 3 shows comparison images from the microtubular network of a human embryonic kidney cell, recorded with the PSF of Fig. 2(a). The microtubules were labeled with Atto532-stained antibodies and the cell was mounted with Vectashield. The images were recorded with a pixel size of $33 \mathrm{~nm}$ along $x$ and $100 \mathrm{~nm}$ along $y$ and a pixel dwell time of $60 \mathrm{~ms}$. The raw data of the GSD recording [Fig. 3(b)] resolves features of the network along the $\mathrm{x}$ axis that are hidden in the confocal reference image. This is further highlighted in the line profiles of Fig. 3(d), where microtubular strands at distances $<80 \mathrm{~nm}$ can be resolved by GSD but not by confocal microscopy.
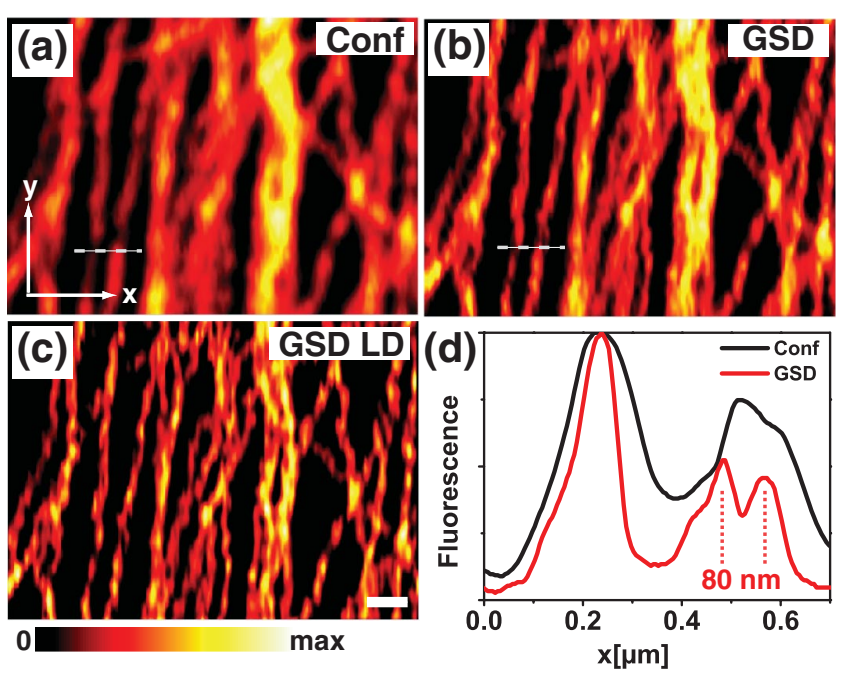

FIG. 3 (color). GSD microscopy provides focal plane resolution $\leq 80 \mathrm{~nm}$ in biological imaging, demonstrated with Atto532-stained microtubules: (a) confocal, (b) GSD, and (c) GSD plus linear deconvolution (LD). (d) Normalized line profiles through the data of (a) and (b) where indicated by the dashed line; images were smoothed using a Lagrangian interpolation of third order. Scale bar $=500 \mathrm{~nm}$.

The dynamics of triplet state saturation, in particular the incomplete $S_{0}$ depletion [Fig. 1(a)], lead to PSFs with a sharp tip and a broader baseline (Fig. 2) implying that in addition to the super-resolution spatial frequencies, the raw GSD data contain a substantial amount of low frequencies. Applying a single-step linear deconvolution (e.g., a Wiener filter) enhances the high frequency content in the image and hence the structural details [Fig. 3(c)].

Next, we applied the doughnut to fluorescent beads of $40 \mathrm{~nm}$ diameter (orange beads, Molecular Probes, Eugene, OR) with peak emission at $560 \mathrm{~nm}$. We attached the beads to a microscope cover slip using poly-L-lysine and covered them with Vectashield. Figure 4 shows comparison images for confocal and GSD microscopy. Both images were subject to a single-step linear deconvolution for fair comparison. The scanning step size was $25 \mathrm{~nm}$ along the $x$ and the $y$ directions and a pixel dwell time of $60 \mathrm{~ms}$. While being blurred in the diffraction-limited confocal image, GSD microscopy separates the beads in all directions. The image pair of Figs. 4(c) and 4(d) demonstrates that the subdiffraction resolution in both lateral directions can also be obtained in biological imaging. Figures 4(c) and 4(d) visualize the spatial distribution of Atto532-labeled SNAP-25 proteins on the plasma membrane of a fixed neuronal cell mounted in Vectashield. As a part of the SNARE family, the protein SNAP-25 codetermines the plasma membrane site at which a vesicle may dock or fuse. Again, the GSD image exhibits a much more detailed view of the protein spatial order than in confocal microscopy.

Exploiting the saturation of a metastable dark state, GSD microscopy entails intensities as low as a few $\mathrm{kW} / \mathrm{cm}^{2}$. 

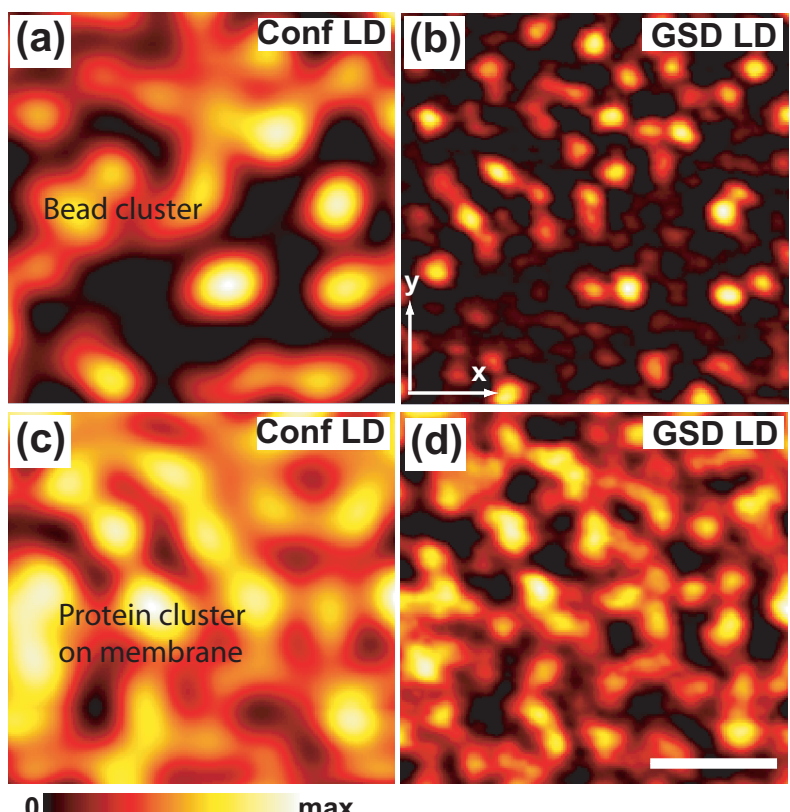

0

FIG. 4 (color). GSD microscopy provides super-resolution in the focal plane. Orange fluorescent beads recorded confocally (a) and with GSD (b). SNAP-25 protein agglomerations stained with Atto532 on a cell membrane: confocal (c) and GSD (d). All images after linear deconvolution (LD). Scale bar $=500 \mathrm{~nm}$.

This benefit obviously stems from the millisecond lifetime $\tau_{T}$ of the dark state. One might conclude that the low intensity operation is inevitably bought at the expense of longer recording times. However, the low light levels utilized in GSD microscopy will facilitate camera-based parallelized subdiffraction imaging with an array of intensity zeros, formed as patterns or lines $[5,6]$, thereby significantly cutting down the recording time.

Photobleaching is known to involve transient dark states, especially the excited levels of these states, as starting points $[10,11]$. Therefore, the selection of dye-media combinations entailing low photobleaching is mandatory. We surprisingly found effective optical shelving in rather different fluorophore classes, such as atto-, rhodamine-, cyanine-, fluorescein-, bodipy- and alexa dyes, and also in different media, such as Vectashield, poly-vinyl-alcohol, and aqueous solvents, as detailed in the auxiliary material [14]. Optimizing the fluorophores and the mounting media should lead to further improvements in GSD performance. Because of increased spin-orbit coupling, the presence of heavy atoms, such as chloride or bromide, should increase the triplet population $[14,15]$, while additional exposure to near-infrared light may shorten the triplet lifetime by reverse intersystem crossing [16]. Heavy-atom doped fluorophores are expected to facilitate GSD microscopy under aqueous conditions. A different option is to apply low temperatures for increasing both the $T_{1}$ lifetime and the photostability.
We note that the fluorophores could be shelved in any other dark state other than $T_{1}$, including radical states $[11,17]$, provided that they return to $S_{0}$. Intriguingly, Eq. (1) implies that optimizing the operational parameters toward $I_{D}^{\max } \gg I_{S}$ enables a spatial resolution, in principle, down to the molecular scale. Combined with general presence of dark states in all fluorophores, our findings underscore the potential to break the diffraction barrier by transient optical shelving.

We thank A. Schönle for help with the measurement software, S. Jakobs and S. Löbermann and J. J. Sieber and $\mathrm{T}$. Lang for help with the sample preparation of the microtubule network and SNAP-25, respectively, and B. Rankin for careful reading of the manuscript. We also thank $J$. Widengren and A. Chmyrov (KTH Stockholm) for valuable discussions. This project was funded by a EU grant (New and Emerging Science and Technology, SPOTLITE).

*Email address: shell@gwdg.de

[1] E. Abbe, Archiv f. Mikr. Anat. 9, 413 (1873).

[2] S. W. Hell and J. Wichmann, Opt. Lett. 19, 780 (1994).

[3] S. W. Hell, Nat. Biotechnol. 21, 1347 (2003).

[4] M. Hofmann, C. Eggeling, S. Jakobs, and S. W. Hell, Proc. Natl. Acad. Sci. U.S.A. 102, 17565 (2005).

[5] R. Heintzmann, T. M. Jovin, and C. Cremer, J. Opt. Soc. Am. A 19, 1599 (2002).

[6] M. G. L. Gustafsson, Proc. Natl. Acad. Sci. U.S.A. 102, 13081 (2005).

[7] E. Betzig, G. H. Patterson, R. Sougrat, O. W. Lindwasser, S. Olenych, J.S. Bonifacino, M.W. Davidson, J. Lippincott-Schwartz, and H.F. Hess, Science 313, 1642 (2006).

[8] M. J. Rust, M. Bates, and X. Zhuang, Nature Methods 3, 793 (2006).

[9] M. Kasha, Radiat. Res. Suppl. 2, 243 (1960).

[10] R. Zondervan, F. Kulzer, M. A. Kolchenko, and M. Orrit, J. Phys. Chem. A 108, 1657 (2004).

[11] J. Widengren, A. Chmyrov, C. Eggeling, P.-A. Löfdahland, and C. A. M. Seidel, J. Phys. Chem. A 111, 429 (2007).

[12] S. W. Hell and M. Kroug, Appl. Phys. B 60, 495 (1995).

[13] S. W. Hell, in Topics in Fluorescence Spectroscopy, edited by J. R. Lakowicz (Plenum, New York, 1997).

[14] See EPAPS Document No. E-PRLTAO-98-005720 for auxiliary data on the resolution of a single point scanning GSD microscope and on the comparison of different fluorophores and mounting media for GSD microscopy. For more information on EPAPS, see http://www.aip.org/ pubservs/epaps.html.

[15] F. Köhn, J. Hofkens, R. Gronheid, M. Van der Auweraer, and F. C. de Schryver, J. Phys. Chem. A 106, 4808 (2002).

[16] S. Reindl and A. Penzkofer, Chem. Phys. 211, 431 (1996).

[17] R. Zondervan, F. Kulzer, S. B. Orlinskii, and M. Orrit, J. Phys. Chem. A 107, 6770 (2003). 